\title{
Thermal load distribution on the ALT-II limiter of TEXTOR-94 during RI mode operation and during disruptions
}

\author{
K.H. Finken, T. Denner, G. Mank \\ Institut für Plasmaphysik, Forschungszentrum Jülich GmbH, \\ Euratom Association, Trilateral Euregio Cluster, Jülich, Germany
}

\begin{abstract}
Thermographic measurements using an IR scanner have been performed at the pump limiter ALT-II of TEXTOR-94 during RI mode discharges and during disruptions. The measurements on the RI mode discharges were done to complete the TEXTOR database which had shown a structured decay pattern of the deposited power. It was found that the underlying radial heat flux can be described by two exponential decay functions. This structure, which generates an unexpected heat component close to the tangent line, has been observed in all discharge conditions including the RI mode. During disruptions, the heat is released in short pulses with a typical duration of $0.01-0.1 \mathrm{~ms}$. The radial decay length of these pulses has a similar shape to the heat flux during normal discharges: it consists again of a strong component close to the tangent line with a radial decay length of $2-5 \mathrm{~mm}$ and probably one with a decay length of the order of $1 \mathrm{~cm}$. The heat is released at the time when the edge electron temperature of the plasma drops, when intense hydrogen and carbon fluxes occur near the walls, and when electrical currents in the limiter blades are excited. In a tentative interpretation, the temporal and spatial structure of the heat pulse is attributed to the presence and growth of a laminar zone at the plasma edge, which is connected with the ergodization of the plasma edge during a disruption.
\end{abstract}

\section{Introduction}

This article deals with the analysis of thermographic measurements on the toroidal ALT-II pump limiter of TEXTOR-94 during RI mode conditions and during disruptions. The first topic is a continuation of recently published work [1] on ohmic and L mode discharges, while the second topic treats predominantly the time dependence of the heat pulses during disruptions in relation to other signals. We take particular advantage of the improvements made to our infrared scanner, which now allows measurements with very high time resolution [2] (order of microseconds).

The observed power deposition pattern on various devices [3-8] has been discussed in Ref. [1] and has been compared with the observations made on TEXTOR-94; therefore no discussion will be given here. The essential result of the previous analysis was that the radial dependence of the heat flux to the limiter cannot be described by a simple exponential law: a good fit can be given by two exponential functions where the one with the shorter decay length dominates close to the tangent line of the limiter with respect to the plasma and the one with the longer decay length dominates further away from the tangent line. The shorter decay length amounts to $1 \mathrm{~mm} \leq \lambda_{\text {short }} \leq 3 \mathrm{~mm}$ and the longer one to about
$8 \mathrm{~mm}$; the power is roughly equally distributed to the components with shorter and longer decay lengths. Probes or atomic beams measuring away from the limiter give results consistent only with the longer decay length. In our opinion, the short decay length near the tangent line may be imposed by the magnetic pre-sheath on a surface with an extremely small angle of incidence of the magnetic field lines (about $1^{\circ}$ ); the details, however, are not yet understood. If this picture of enhanced heat flux can be transferred to the target plates of divertors, the power load may change relative to the picture anticipated up to now. We believe that the effect should be considered for the layout of divertor target plates of the next step device.

To obtain the information quoted above, the following steps had to be taken: the temperature evolution along a poloidal arc of the graphite tiles must be recorded and serves as input data for calculating the heat flux $Q(\vartheta)$ by a code which takes into account, in particular, the tile thickness at the different locations. The poloidal arc co-ordinate represents also the radial co-ordinate because, when progressing poloidally, the tiles bend away monotonically from the tangent line. The radially resolved power $P(r)$ is then given by

$P(r)=\frac{Q(\vartheta(r))}{\boldsymbol{n} \cdot \boldsymbol{b}}$ 


\section{K.H. Finken et al.}

where $\boldsymbol{n}$ is the vector normal to the limiter surface and $\boldsymbol{b}$ is the unit vector in the direction of the magnetic field.

The surface normal vector is obtained from knowledge of the tile geometry; as the surface normal vector is nearly orthogonal to the local magnetic field unit vector (the angle amounts to about 86$89.4^{\circ}$ ), many details of the magnetic field have to be taken into account such as $I_{p}, \beta_{\text {pol }}$ and plasma shift. Even though TEXTOR-94 has a relatively small magnetic field ripple due to the relatively large distance between the TF coils and the plasma, this ripple imposes dominant modifications to the heating power pattern. An important feature resulting from the ripple is the self-shadowing of the field lines, which creates a private flux zone of short connection length (field lines entering the limiter surface, then leaving it for a short distance and intersecting it again).

Up to now, the power distribution during disruptions has been less thoroughly analysed than the one of normal discharges. It had already been described earlier [9] how during disruptions the energy is released typically in the form of short spikes and not smoothly over the whole current decay phase. The development of disruptions has been treated by several authors [10-28]. It is widely accepted that a disruption is often initiated by precursor modes, in particular those at the $q=1, q=1.5$ and $q=2$ surfaces. At present, it is unclear whether the modes are growing such that the overlap criterion is always fulfilled and the plasma interior becomes ergodized. After this initiation phase, the proper disruption sets in with a negative loop voltage spike and the plasma current decay phase which lasts for typically some $10 \mathrm{~ms}$. The sudden loss of plasma energy occurs at the time of the negative spike in the loop voltage. This energy loss appears at plasma facing components as enhanced heat flux. It can lead to a release of impurities from the walls, which then cool the plasma and accelerate the decay phase [29].

The disruptions are often categorized according to the violation of limits of stable tokamak operation [30]: density limit disruptions, q-limit disruptions, loss of position control (or too large a plasma shift) or $\beta$-limit disruptions. The underlying physics for the development of disruptions is often an unstable distribution of the plasma current (e.g., no current outside of the $q=2$ surface due to $q(a) \approx 2$ ( $q$ limit, position control), cooling of the edge (density limit) or unfavourable internal current distribution $(\beta$ limit)). These current distributions generate growing kink modes and/or tearing modes [31-39], which become dangerous particularly if the modes lock [40-47]. Recently, it has been proposed to avoid the critical error field effects [48] by constructing a 'fake shell' [49, 50]. On TEXTOR-94, a stabilizing coil system is foreseen within the project of the Dynamic Ergodic Divertor (DED) [51] acting either by imposing a torque [52] on the plasma or as a feedback system.

In this article, the time dependence of the heat pulses and its relation to other events during the disruption will be described. The data may be of importance for designing plasma facing components for a next step device. It is not intended in this article to analyse the development of the plasma interior during a disruption.

\section{Set-up}

TEXTOR-94 is equipped with a nearly full toroidal pump limiter located at the outboard side $45^{\circ}$ below the equatorial midplane. The limiter consists of eight individually movable Inconel blades which are covered with 28 graphite tiles each. A detailed description of the ALT-II limiter can be found in a special issue of Fusion Engineering and Design [53]. To optimize the particle exhaust during the discharge, the projected radial thickness of the pump limiter should be small; the thickness, however, is limited by the requirements due to the incident heat flux.

At present, ALT-II is covered with a set of graphite tiles with a projected radial thickness of about $20 \mathrm{~mm}$, as was discussed in Ref. [1]. In contrast to the older tile versions, these tiles have no central tangent surface section; they have been laid out to sustain $8 \mathrm{MW}$ of heating power for $10 \mathrm{~s}$. The end tiles consist of felt type carbon-carbon composites (CX-2002U) with enhanced radial and poloidal heat conductivity while the rest of the tiles is made from fine grain isotropic graphite (IG-430U). The tiles are shaped toroidally, and the angle of incidence of the magnetic field lines is smaller than $1^{\circ}$ in the first $10 \mathrm{~mm}$ of the SOL (see also Fig. 2(b)). The blades have roof type shape; the area around $45^{\circ}$ is parallel to the magnetic flux surface for about $3 \mathrm{~mm}$ (in the poloidal direction) and then starts to deflect from there with slowly increasing angle of incidence of the magnetic field lines. The tile design would provide a rather uniform heating of the blades if the power $e$ folding length amounts to $7 \mathrm{~mm}$ (as measured earlier [8]) and if the magnetic field ripple is neglected. 
The IR scanner (Inframetrix model 760) views the pair of tiles next to the end tiles. This set of tiles was selected because

(a) It avoids the end tiles, which are more strongly heated than the rest (due to a gap of $15 \mathrm{~cm}$ in between two blades).

(b) The location allows us to interpret the data if the magnetic field ripple is taken into account (Fig. 4 of Ref. [1]).

(c) An observation port could be made available.

The tile shape is in particular suited to determine the radial profile of the incoming power near the last closed flux surface, i.e. the fraction of deposited power with small decay length. The spectral range of the camera extends from 3 to $8 \mu \mathrm{m}$ where the lower limit is mainly determined by the transmission of the germanium entrance optics and the upper limit by the $\mathrm{CaF}_{2}$ window and lenses we used.

To understand the measurements on disruptions, a few more details of the IR scanner have to be discussed. The basic elements of the scanner are a horizontally sweeping mirror, a vertically sweeping mirror and the IR detector. The detector itself provides only time and no spatial information such as a CCD chip would do; the spatial information of the system is introduced by the scanning mirrors. The vertical mirror moves in a sawtooth-like manner and sweeps over the picture within $20 \mathrm{~ms}$ just as given by the TV standard. The horizontal movement of the mirror is too fast for the sawtooth-like characteristic; therefore only a harmonic motion is performed, one line from right to left and the next one from left to right. For fast events, the scanner provides the option to operate on ' $8 \mathrm{kHz}$ (line scan) mode' by switching off the vertical mirror motion. This option restricts the spatial information in order to gain higher time information. With respect to time evolution, the IR scanner data are analysed from frame to frame (time resolution $20 \mathrm{~ms}$ ), from the top of the frame to the bottom (time resolution about $100 \mu \mathrm{s}$, given by the time needed for a line) and finally within a line (time resolution microseconds).

To obtain such detailed time information and other improvements, the internal electronics of the IR scanner were substantially altered [2]:

(a) The time correlation was obtained by adding a quartz clock and by careful bookkeeping of odd and even TV lines. This procedure allows one to attribute a time to each point of the image. The clock of the scanner and the clock from the data acquisition are synchronized at the start of the discharge but run independently. It is therefore not surprising that the heat pulse and particle fluxes occur at the same time in disruptions early in the discharge, while a systematic shift is observed for disruptions occurring later in the discharge.

(b) The dynamic range was raised from 7-8 bits to 12 bits. The high dynamic range thus has an extremely high value for the disruptive events with the highest power loads.

(c) The data transfer is fully digital with fibre optics links only. This modification avoids any electrical loop which may be sensitive for inductive voltage pick-up in particular during disruptions. The scanner and the electronic boxes are carefully shielded by iron, $\mu$-metal and copper against all kinds of magnetic or electrical disturbances.

The high time resolution as required for disruptions (microseconds) can in practice only be utilized if additional conditions on the object under observation are fulfilled: if the temperature distribution of the object is smooth a sudden change can easily be recognized. If, however, the temperature distribution is non-uniform before the arrival of a heat pulse, a detailed analysis is very difficult. For the analysis of the disruptive heat load, the line scan mode was therefore applied. Even then we had to select some rare disruption events where the heat pulse was released when the scanner was recording the temperature at a smooth part of the limiter. An additional constraint for the analysis of the data (if one is using a scanner instead of a fast diode which was not available) is the temperature consistency in the frames and lines before and after the power pulse. These observed temperature rise times in these few disruption cases have been taken as typical for the disruptions and are quoted in Section 4.2.

Figures $1(\mathrm{a}-\mathrm{d})$ give an overview of the viewing field and some characteristic IR images. Figure 1(a) is a view through the IR window with a camera operating in the visible spectral range. In the background, one sees the TEXTOR liner with some characteristic holes for the orientation. One ALT-II blade extends from the middle of the figure to the bottom. The graphite tiles on the blade are labelled by numbers, which are shown next to them. The subfigures are tilted with respect to the proper arrangement in TEXTOR: the top row of tiles is formed by the tiles $1-14$ and the bottom row by tiles $15-28$. If a line 


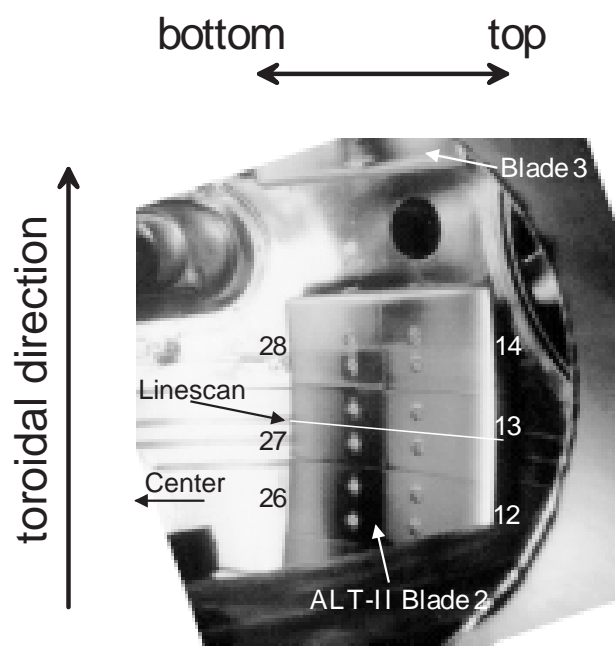

(a) View through IR-Window

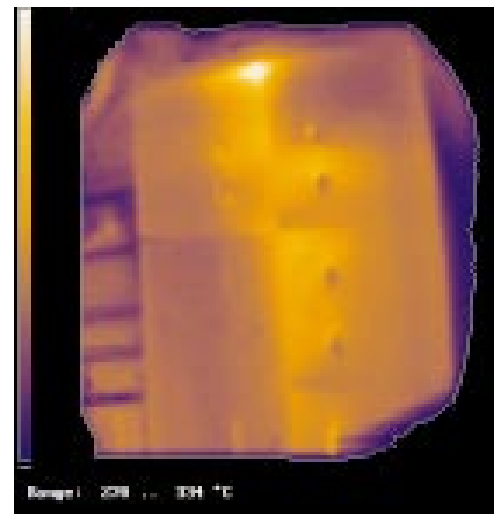

(b) Image Mode (\#75423 @2,64 s)

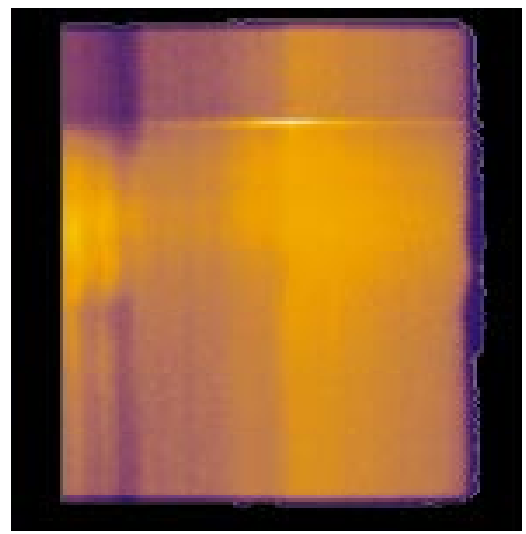

(c, d,) Fast Line Scan Mode (8000 lines/s, one frame corresponds to $20 \mathrm{~ms}$ )

\# 81539: normal discharge

\# 81540: disruption

Figure 1. (a) View through the IR window towards the observed ALT-II tiles; the picture is taken with a normal camera in the visible. (b) Typical IR image of the ALTII tiles during a discharge. (c) Thermographic picture of the centre of tiles 13 and 27 taken in fast line scan mode (only the horizontal mirror is sweeping). (d) Disruption taken in fast line scan mode (shot 81540). The IR scanner has been oriented in the picture such that the toroidal direction is from top to bottom; in this set-up line scan images along the poloidal arc, as indicated in (a) by the horizontal line, can be performed.

scan is performed, its location is selected such that it occurs in between the bolts which fix the tiles to the baseplate. Figure 1(b) displays the IR image during a discharge. As explained above, the top tile (tile 13 ) is more heated than the bottom tile (tile 27). From the temperature distribution along a poloidal arc, the radial power deposition pattern is derived. Figure 1(c) shows a normal discharge in the line scan mode, the scan line being chosen in the centre of tiles
13 and 27 as indicated in Fig. 1(a). Figure 1(d) shows the occurrence of a disruption again in the line scan mode. The time increases from the top to the bottom in the frame. The disruption is the event where the false colour is suddenly changing within one line. After a disruption, one may see modulations of the heated parts due to the shaking of the vacuum vessel of TEXTOR and a mirror which is attached to it. The oscillation is strongly damped. 
Article: Thermal load distribution on ALT-II

\section{Power flux distribution during RI mode discharges}

Radiative improved (RI) discharges are the high confinement discharges in TEXTOR-94 [54]. The seeding of medium- $Z$ impurities (neon, argon, silicon, etc.) in the plasma edge produces a steepening of the plasma density profile. In contrast to other regimes of high energy confinement, the RI mode improves with increasing density such that optimum confinement values are obtained close to the Greenwald limit; there the confinement quality is as good as that of the ELM-free $\mathrm{H}$ mode. For plasma heating, typically NBI (preferentially co-injection) and ICRH are applied.

The power put into the plasma is predominantly radiated away from the boundary zone of the plasma. The radiation fraction is larger than $60 \%$, going up to $95 \%$; the remaining power fraction conducted to the limiters amounts to only $40 \%$, down to $5 \%$, of the input power, respectively. The power fraction in L mode discharges amounts to typically $40-60 \%$ at those high densities. In this context it is of interest, whether the radial deposition profile of the power is modified in the high confinement regime with the high radiative power fraction at the edge or not.

Figure 2(a) shows an example of the temperature distribution along a selected poloidal arc and Fig. 2(c) the resulting power flux along the magnetic field. The top tile extends from 28 to $45^{\circ}$, while the bottom one is located between 45 and $62^{\circ}$. As we had already discussed previously [1], the temperature on the top and bottom row of the graphite tile is not simply symmetric around the tangent line in the centre of the blade $\left(45^{\circ}\right)$ even though the plasma is well adjusted. The problem arises from the scalar product $\boldsymbol{n} \cdot \boldsymbol{b}$ of the surface normal vector with the magnetic field vector, which contains field ripple as an additional complication [1]. This field ripple leads to a self-shadowing of the incoming power flux (private flux zone with a connection length of the order of one metre). This self-shadowing occurs on the bottom tile, resulting consequently in a smaller temperature rise than at the top tile. Therefore, the evaluation of the radial power flux distribution is meaningful for the top tile (tile 13) only. The analysis of all IR pictures was taken along the line indicated by 'line-scan' of tile 13 in Fig. 1(a).

Figure 2(b) shows the angle of incidence of the magnetic field lines on the surface of the top tile (tile 13), which is investigated in detail. The angle of incidence is very shallow, even for plasma facing
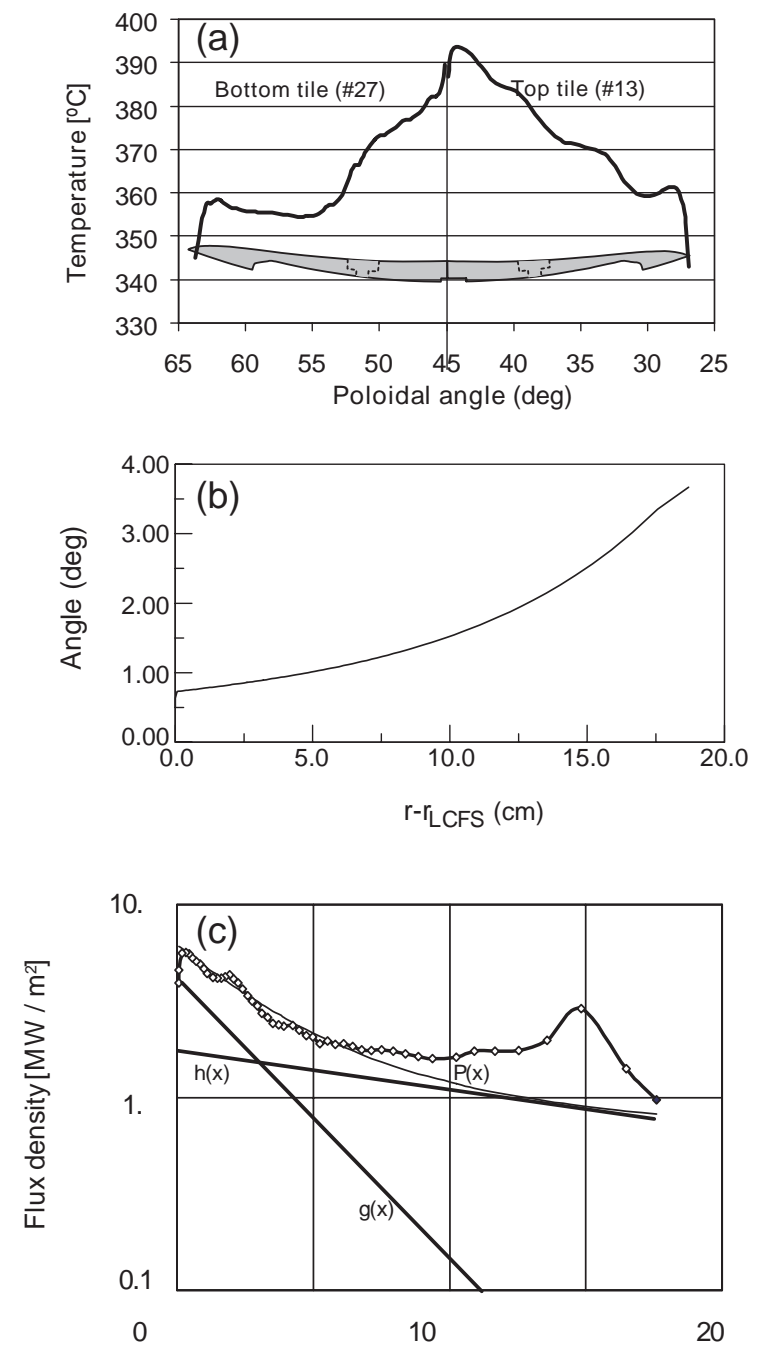

Radial distance in the SOL from the last closed flux surface [mm]

Figure 2. (a) Temperature distribution along the poloidal arc of tiles 13 and 27 during an RI mode discharge. (b) Angle of incidence of the magnetic field lines on the surface of tile 13 for the line scan cut shown in Fig. 1(a). (c) Radial power deposition function deduced from the temperature development.

components; it varies between $0.7^{\circ}$ near the tangent line of the limiter to $3.6^{\circ}$ at the entrance to the pumping scoop (remote part of the tile).

The observed temperature distribution on the tile surface is not completely smooth; there is some expected modulation due to the variation of the tile thickness (recessed graphite volume for mounting the tiles on the Inconel blade). There is, however, also some unexpected variation of the temperature, in particular at the edge of the tile (e.g. around poloidal angles of 30 and $60^{\circ}$ ). This enhanced temperature leads to the flux enhancement in Fig. 2(c) at a radial 


\section{K.H. Finken et al.}

distance of about $15 \mathrm{~mm}$. A closer inspection of the IR recording indicates that this maximum is most likely due to reflections at the tile surface: the surface is bending away from the direction from the optical axis such that the surface appears at a glancing angle. It seems that some parts of the liner or wall are strongly heated from the intense radiation of the RI mode discharge and that the above mentioned part of the tile surface reflects this light into the IR optics - simple estimates yield a temperature rise of the liner up to $100^{\circ} \mathrm{C}$ due to the radiation in strongly heated RI mode discharges.

We were not able to identify any other reasonable causes of the enhanced temperature at the remote part of the limiter tiles. Effects like magnetic field ripple have been carefully investigated because they are essential for the analysis of the radial decay function of the power; the field ripple creates a characteristic heating pattern, which is described in detail in Fig. 4 of Ref. [1]. The heating pattern is a combined effect of the power decay function away from the last closed flux surface and the angle of incidence with respect to the tile surface. In this figure, the TF coils are located in between two blades (i.e. right and left of the figure) and in the middle of the blades (i.e. between tiles 7,8 and 21, 22). For the given tile shape of ALT-II, the heating pattern shows four maxima per blade, two on the upper row (i.e. tiles 6 and 13) and two on the lower row (tiles 16 and 24). The maxima are nearly top-bottom symmetric, the top maxima are shifted toroidally versus the bottom ones, however, they neither occur on the maxima of the field line deflection nor just in between where the radial field component is maximum. The heating pattern is predominantly determined by the conventional cosine dependence of the angle of incidence; nevertheless, normal intuition can easily guide in a wrong direction.

Because the heating pattern at the outer limiter edge is well described for all discharge conditions other than those of the RI mode by the conventional cosine dependence of the angle of incidence, we see no reason to assume that the cosine law should be violated. In particular, the otherwise similarly heated $\mathrm{L}$ mode discharges show the expected temperature dependence.

The power flux density along the magnetic field, i.e. the flux density which would fall on an object oriented perpendicularly to the field lines, is plotted in Fig. 2(c). The value amounts to about $8 \mathrm{MW} / \mathrm{m}^{2}$; when only looking at the numbers, this flux is of similar size to the one treated during the disruptions.
However, the disruptive heat flux will not be projected along the magnetic field (because of too many uncertainties of the exact position of the plasma) but will be the flux on the shallow ALT-II surface, as has been shown in Fig. 2(b). To compare the fluxes, one has to take into account the cosine of the angle of the magnetic field line with the surface.

As in the previous publication [1], it was tried to fit the flux density function of Fig. 2(c) again with two exponential functions (neglecting the excessive heat around $\Delta r=15 \mathrm{~mm}$, which we attribute to reflections); we subtract at first the radiative fraction of the tile heating which we obtain from bolometric measurements $\left(0.05 \mathrm{MW} / \mathrm{m}^{2}\right)$. This procedure leads to decay lengths of $\lambda_{\text {short }} \approx 2.5-3 \mathrm{~mm}$ and $\lambda_{\text {long }} \approx 40 \mathrm{~mm}$. The short decay component agrees with previous results from other modes of operation and, as comparison with Fig. 2(b) shows, it is observed for an angle of incidence of less than $1^{\circ}$. The value of the long decay length is a factor of four to six larger than observed previously. Integrating radially the power fluxes of both components would yield a four times higher power flux to the long decay length component. This result, however, is not compatible with the observed particle removal by the pump limiter ALT-II: The decay length for the temperature, $\lambda_{T}$, and that for the density, $\lambda_{n}$, are connected with the decay length for the particle fluxes $\lambda_{\Gamma}$ and that for the power flux, $\lambda_{Q}$, by $\lambda_{\Gamma}=2 \lambda_{n} \lambda_{T} /\left(2 \lambda_{T}+\lambda_{n}\right)$ and $\lambda_{Q}=2 \lambda_{T} \lambda_{n} /\left(2 \lambda_{T}+3 \lambda_{n}\right)$ [55]. If the RI mode could create such a strong component with long $e$ folding length in the power decay length, particle removal would drastically increase in the transition from $\mathrm{L}$ mode to RI mode. This, however, is not observed; the removal rate decreases by about the same factor as $\tau_{p}$ decreases. Therefore, we have to assume that the power in the component with the long decay length is about the same as that with the short decay length, just as in L mode and in ohmic conditions. If we perform such a fit we have to add a constant term and obtain as a best fit,

$$
\begin{aligned}
& P(x)\left[\mathrm{MW} / \mathrm{m}^{2}\right]=g(x)+h(x) \\
& g(x)=4.4 \exp (-x / 3) \\
& h(x)=1.2 \exp (-x / 10)+0.6
\end{aligned}
$$

where $x=r-r_{L C F S}$ is the distance from the last closed flux surface. We attribute the constant contribution in $h(x)$ to the combined effect of additional radiation from carbon impurities and of charge exchange particles. However, we are not able to 


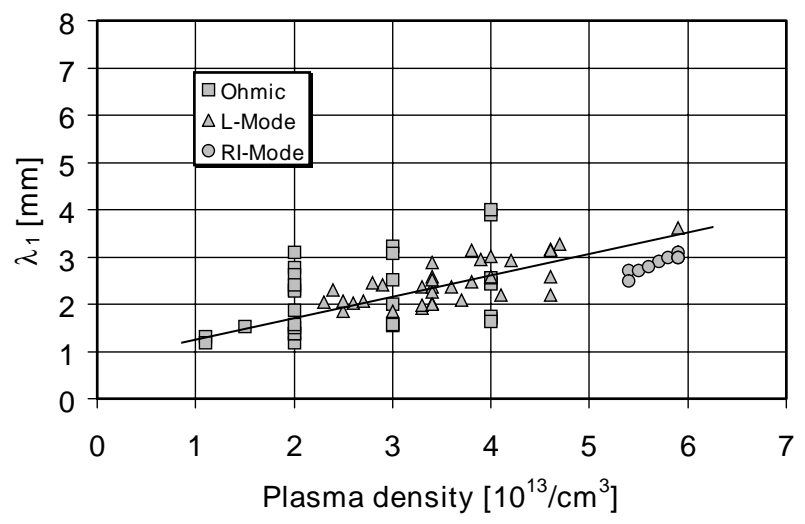

Figure 3. Compilation of the 'short' power decay length fraction $\lambda_{1}$ for different types of discharge mode on TEXTOR-94. The RI mode discharges show essentially the same features as the other discharge types.

model these two effects and their distribution on the tiles. Therefore the determination of the component with the long power decay length has some uncertainties. The fitting functions $P(x), g(x)$ and $h(x)$ are also shown in Fig. 2(c).

A compilation of the 'short' power decay length fraction, which is dominant close to the tangent line, is shown in Fig. 3 for different types of discharges. The RI mode discharges are represented by the circular symbols at highest densities. The data points are slightly below the averaged curve, but considering the scatter of the data points from ohmic and $\mathrm{L}$ mode discharge conditions taken under various discharge conditions, this deviation is not considered to be significant.

The most important information deduced from the measured power deposition is that the enhanced power flow near the tangent line persists for all discharge types on TEXTOR-94. Uncertainties from reflections at the limiter tile may influence evaluation of the component with the long power decay component but they do not affect the component with the short power decay length.

\section{Power flux distribution during disruptions}

\subsection{The conditions}

The disruptions shown in this section stem from one day dedicated to producing high densities (MARFE programme). As disruptions were expected on that day, some boundary signals were recorded with high time resolution for a good correlation amongst the disruption data. These data are the $\mathrm{H}_{\alpha}$ flux to the ALT-II limiter, $\mathrm{H}_{\alpha}$ flux to the inner bumper limiter, CII flux to the inner bumper limiter and electrical currents to the individual ALT-II blades.

Most features described in the following do not depend on the specific type of disruption (e.g. high density limit disruptions, as here) but they are typical of all disruptions. A feature common to all disruptions is the power release from the plasma in the form of short pulses. The absolute amount of energy in a pulse is often observed to be larger for low density disruptions than for high density disruptions; there are also more power pulses in the late phase of the disruption namely during the current decay phase. We have, nevertheless, chosen the high density disruptions and disruptions due to strong mode excitation (those examples occurring in the plasma buildup phase), because the plasma column tends to remain rather well centred immediately at the onset of disruption. This is in contrast, for example, to low density disruptions, which are often caused by loss of plasma control.

\subsection{Temporal and spatial deposition pattern}

As already mentioned, during disruptions the power is released towards the wall components in the form of pulses. An example of such a heat pulse has already been shown in Fig. 1(d), while Fig. 1(c) represents the temperature on the ALT-II surface during the preceding regular discharge. The time 'runs' from top to bottom in these discharges. Shown is a frame with a time span of $20 \mathrm{~ms}$. It is obvious that the temperature of the surface increases very rapidly and also decays again rather quickly. In this case, only a single pulse appeared in the TV frame. However, another singular heat pulse stemming from a minor disruption had already occurred one TV frame ago during the same discharge. A detailed analysis of the heat pulses (this is analysed from the temperature development during a horizontal sweep as pointed out in Section 2) shows that the temperature maximum is already reached $10 \mu \mathrm{s}$ after the onset of the heat pulse. For a $\delta$ function shaped heat flux, the surface temperature would decay as $T_{s} \propto 1 / \sqrt{t}$; this square root type of dependence following a heat pulse is rather well fulfilled. More details of the power flow will be given.

Not only the temporal development of the power release during disruptions is of interest for the layout 


\section{K.H. Finken et al.}

of plasma facing components in a fusion reactor, but also the spatial distribution of the deposited energy. The experimental observations show that the power deposition pattern is never reproducible. In most cases the disruptive power flux is not deposited centrally on the blade as in a normal discharge; preferentially, the edges of the blades are hit. The reason for the non-symmetric power deposition is most probably the loss of position control during a disruption. In addition, the non-symmetry could be caused by modes at the plasma edge winding helically around the plasma column; it would be expected that the phases of these modes are distributed randomly and that the plasma of these modes touches the limiter surface non-symmetrically.

Figure $1(d)$ is one of the few examples where the power deposition maximum occurs around the tangent line in the centre of the blade and therefore this example is presented. The rather symmetric deposition is a pre-requisite for deriving the radial decay length of the deposited power. As stated in the introductory sections, the IR scanner provides both temporal and spatial information; if the temporal changes are either very slow or very fast as compared with the duration of a frame, one can extract both types of information. Here the disruptive power pulse occurs during one line such that the resulting temperature variation can be analysed both poloidally (i.e. radially) and temporally from line to line. When comparing the disruptive temperature distribution of Fig. 1(d) to the one of a normal discharge as displayed in Fig. 1(c), it becomes obvious that the fraction of the power flux with the short decay length near the tangent line is also a prominent feature of the disruptive heat pulse. From our experience on the analysis of heat deposition, we see immediately that the decay length is not much longer (e.g. not more than $50 \%$ ), because otherwise the heating pattern would not be concentrated in the middle of the blade but would be distributed all over, poloidally. The power fraction with the longer decay length can only be analysed with much higher uncertainty. We believe that our old measurements made on the earlier set of tiles are still valid [9]; it was discussed there that the $e$-folding length for power deposition (long decay length fraction) does not increase by more than a factor of two, i.e. $\lambda_{p \text {-long }} \leq 15 \mathrm{~mm}$.

The fact that the power decay pattern is not changing significantly during disruptions as compared with normal discharges is rather surprising. It will be shown that the disruptive power flux density falling on the tiles amounts to more than $10^{7} \mathrm{~W} / \mathrm{m}^{2}$.
Relating this flux $\Phi$ to a diffusive heat flux would yield

$\Phi=10^{7} S_{A L T}=S_{\text {Plasma }} \kappa \frac{T}{\lambda}$.

Here $S_{A L T}$ is the heated surface of the ALT-II limiter $\left(S_{A L T} \approx 1 \mathrm{~m}^{2}\right), S_{\text {Plasma }}$ is the plasma surface area $\left(S_{\text {Plasma }} \approx 30 \mathrm{~m}^{2}\right), \kappa$ is the perpendicular heat conductivity, $T(\mathrm{eV})$ is the plasma temperature just before the heat pulse (assumed to be $50 \mathrm{eV}$ ) and $\lambda$ is the radial decay length $(\lambda \approx 0.01 \mathrm{~m})$. These data would result in a value of the perpendicular heat conductivity of $\kappa \approx 10^{2} \mathrm{~m}^{2} / \mathrm{s}$. Since this disruptive heat conductivity would be about a factor of 100 higher than normal, one would expect an enlargement of the radial decay length by the square root of this factor, i.e. by about ten. This enlargement, however, is not consistent with the observations, and therefore we think that a diffusive heat transport during the heat pulses must be ruled out; the heat flux seems to be aligned closely to the local magnetic field.

\subsection{Correlation of different signals during disruptions}

For the following discussion we have selected four typical disruptions, each one with different features. The first two disruptions have a single and a double heat pulse, while the two others are preceded by minor disruptions shortly before the proper disruption. The first two occur during the current flattop part of the discharge (shot 81532 at $3.1 \mathrm{~s}$ and shot 81523 at $3.3 \mathrm{~s}$ ), while the other two occur at the end of the current rampup phase (shots 81540 and 81533 at $0.5 \mathrm{~s}$ ). Figures $4-7$ display the characteristic features. Figure set (a) (Figs 4(a), 5(a), 6(a) and $7(\mathrm{a})$ ) shows signals often used to characterize a disruption; they are, from top to bottom, density, plasma current, loop voltage and the $\mathrm{H}_{\alpha}$ signal taken from the ALT-II limiter. Figure set (b) shows the temperature development inside the plasma at the locations labelled at the side of the traces; as a time reference the $\mathrm{H}_{\alpha}$ signal taken from the ALT-II limiter is added. Figure set (c) displays hydrogen and carbon fluxes at the ALT-II limiter and at the inner bumper limiter. In addition, it contains the electric currents to blades 1-4 and the surface temperature of the ALT-II limiter obtained from the IR-scanner (precisely: the temperature of one horizontal line scan position near the tangency point versus time). Figure set (d) finally contains again the surface temperature and derived from this the incoming power density and the integrated energy density. The time 
Article: Thermal load distribution on ALT-II

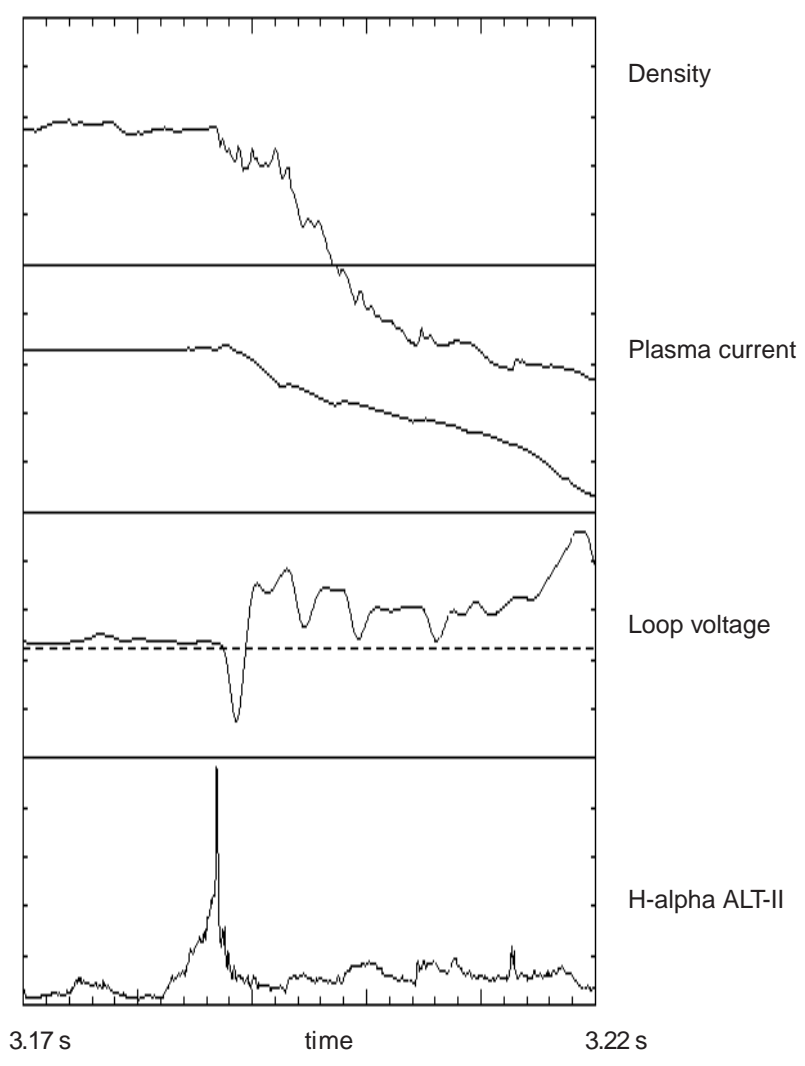

Figure 4(a). Density, plasma current (0-500 kA), loop voltage $(-40 \mathrm{~V}$ to $+50 \mathrm{~V})$ and the $\mathrm{H}_{\alpha}$ signal (arbitrary units) taken from the ALT-II limiter. The density signal is a raw signal; the full scale corresponds to about $7.5 \times$ $10^{19} \mathrm{~m}^{-3}$. Disruption shot 81532 .

axis may vary from subfigure to subfigure depending on the complexity of the individual disruption.

The predisruptive development is best seen in the electron temperature evolution in figure set (b). The settings for $T_{e}$ are chosen such that all signals are plotted nearly equally high; it ranges from $1.5 \mathrm{keV} /$ unit down to $200 \mathrm{eV} /$ unit. The core temperature starts to decrease already well before the negative voltage spike or other characteristic signals. This decrease can set in already $5 \mathrm{~ms}$ before the proper disruption as shown for shot 81532 . While the core temperature decreases, the edge channels show an increase of the electron temperature which suddenly decays when the disruptive heat pulse hits the limiter. The heat pulse and the intense hydrogen flux to the limiter probably occur simultaneously (the small mismatch in the time of the maxima of the signals in figure set (c) is probably due to the mismatch of the clocks as discussed earlier). If multiple heat and particle pulses are released, for example, in the predisruptive phase (more precisely between the minor

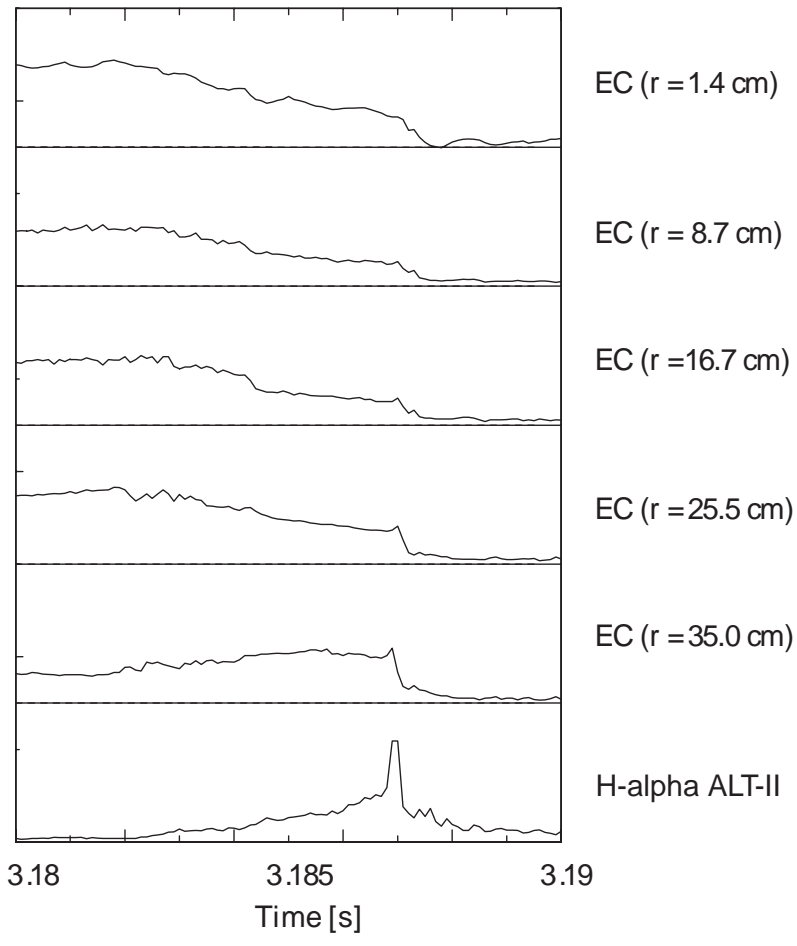

Figure 4(b). Temperature development inside the plasma at the locations labelled at the side of the traces; as a time reference the $\mathrm{H}_{\alpha}$ signal taken from the ALT-II limiter is also shown. The temperature ranges of the EC signals are (from top to bottom) 1.5, 1.5, 1, 0.5, $0.2 \mathrm{keV}$. Disruption shot 81532 .

disruption and the major disruption) as shown in shot 81533 , the recovery of edge temperature precedes each of such pulses. The rather slow decrease of the core temperature of shot 81532 may result from internal modes which may enhance the heat transport inside the plasma. The presence of the modes can also explain the slow increase of the $\mathrm{H}_{\alpha}$ signal at the ALT-II limiter prior to the disruption; these modes may already induce an enhanced transport of particles in the edge.

The characteristic disruption signals of figure set (a) bear no surprise: the negative loop voltage spike marks the start of the current decay phase, which lasts for about $20 \mathrm{~ms}$. The particle flux towards the limiter seems to occur just before the negative voltage spike; however, it is not clear how reliable this statement is because the loop voltage has not been recorded with as high a time resolution as the fluxes.

The particle and heat fluxes and the electric currents to the blades are displayed in figure set (c). The $\mathrm{H}_{\alpha}$ signal at ALT-II occurs typically just prior to the $\mathrm{H}_{\alpha}$ fluxes or carbon fluxes at the inner bumper limiter (e.g. shot 81523). This result may be surprising 


\section{K.H. Finken et al.}

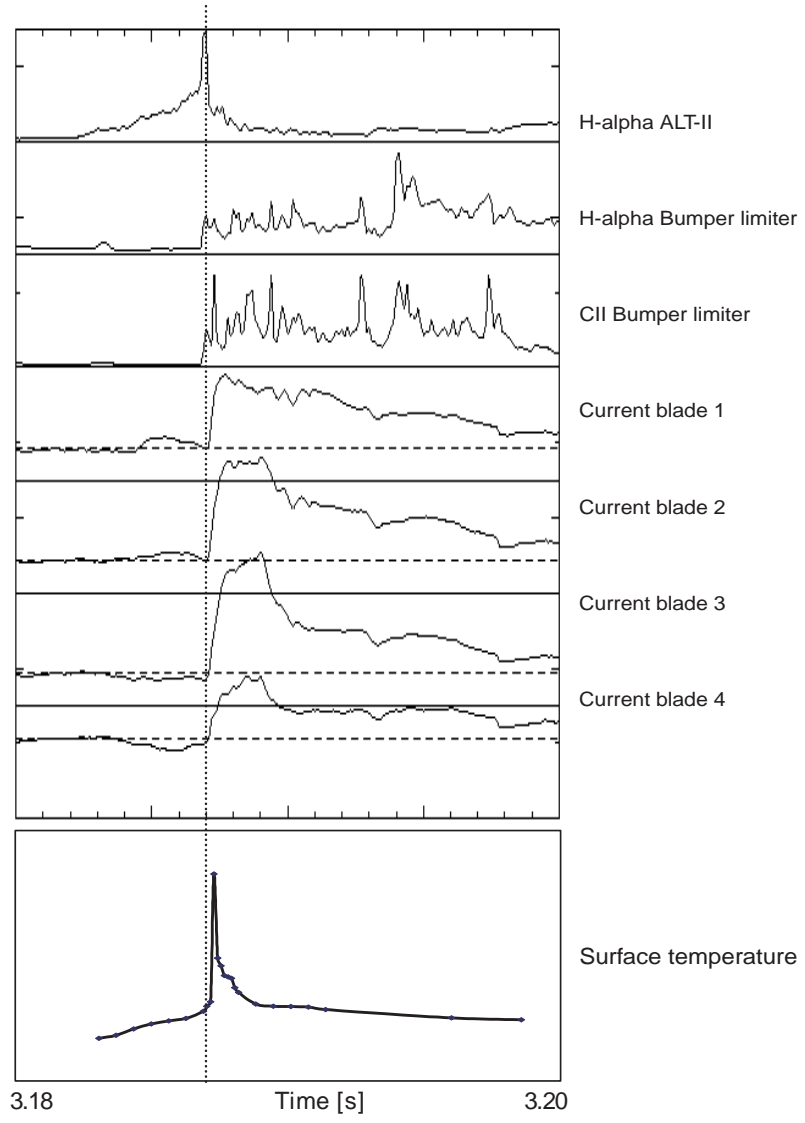

Figure 4(c). Hydrogen and carbon fluxes at the ALT-II limiter and at the inner bumper limiter. In addition, the electric currents to blades 1-4 and the surface temperature of the ALT-II limiter obtained from the IR scanner are also shown. The scale of the limiter currents ranges from -20 to +50 A. Disruption shot 81532 .

because it is often stated that the plasma column shrinks due to the loss of internal energy and that therefore the plasma deposits its energy at the high field side wall. For a limiter tokamak, this statement is obviously not always true; the reason may be that the plasma was in contact with the outboard limiter before the disruption started and because this limiter is closest to the plasma column, it is hit first by the disruptive heat flux. The relative intensity of the different flux signals is widely varying, probably because the disruptive heat and particle fluxes are not toroidally or poloidally symmetric. High fluxes of hydrogen and carbon are seen all over the current quench phase, while disruptive heat pulses so late in the disruption were seen very rarely in this series of discharges.

During normal discharges, the electric current in the individual blades has the same negative sign for

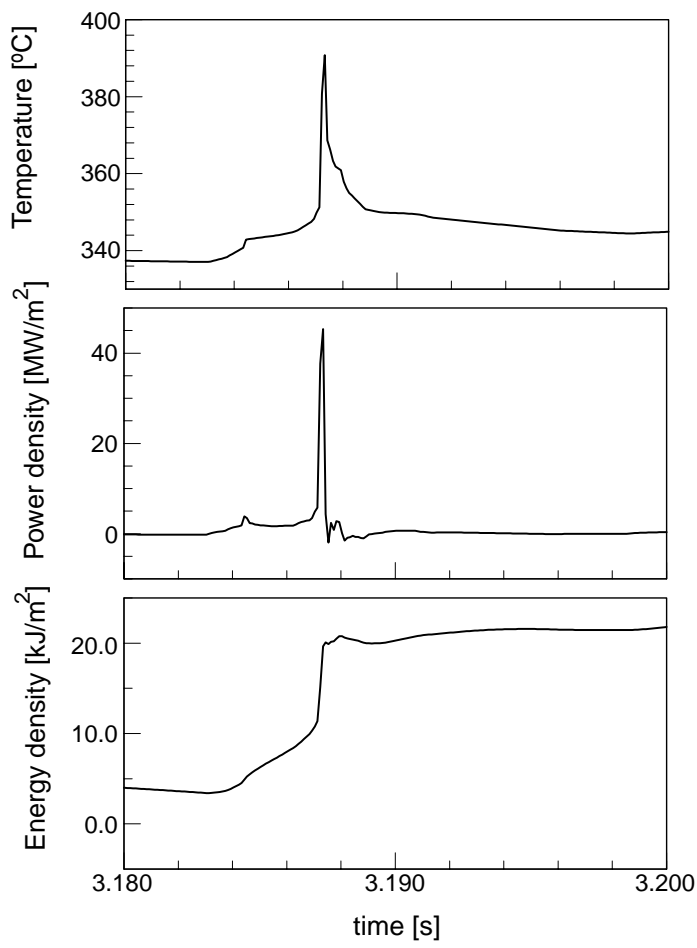

Figure 4(d). Surface temperature and, derived from this, the incoming power density and the integrated energy density. Disruption shot 81532 .

all blades if they are well aligned. The total current varies from 20 to $100 \mathrm{~A}$ depending on the conditions. This current must have a return path which ends most probably at the inner bumper limiter. This current, however, is not measured. With the onset of the disruptive heat pulse, the sign of the current changes and its amplitude is highly increased. The current is observed throughout the current quench phase. The presence of the current is a sign of intense contact of the plasma with the limiter even though no particularly strong heating of the blade is observed. The onset of the disruptive limiter current is not toroidally symmetric; it seems more reasonable to assume that a toroidally rotating structure touches the blades one after the other. This structure may be the deformation of the plasma column due to modes located near the edge of the plasma which rotate toroidally. The heating power of the electric current (typically $50 \mathrm{~A} /$ blade at $50 \mathrm{~V}$ ) is relatively small as compared with the heat release from the plasma (Section 4.4). In addition, this power is predominantly in the plasma current decay phase, i.e. well behind the heat pulses. 
Article: Thermal load distribution on ALT-II

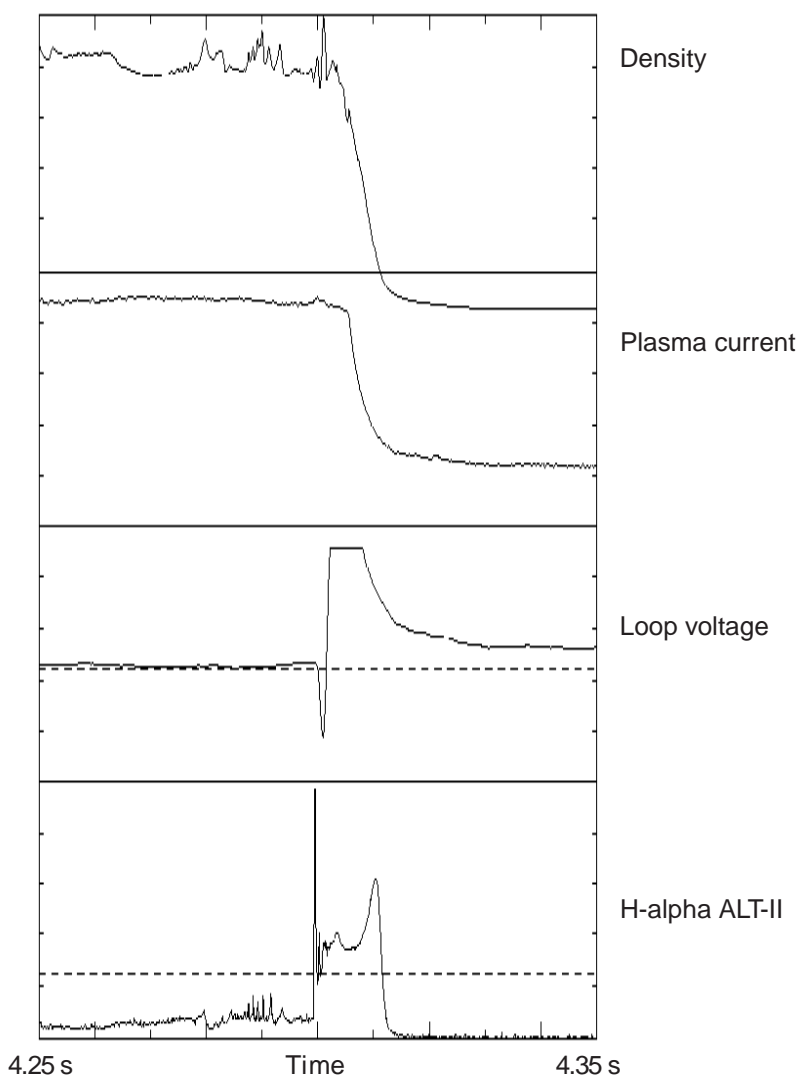

Figure 5(a). As Fig. 4(a) but for disruption shot 81523. The loop voltage measurement saturates at $50 \mathrm{~V}$ (flat part of the curve).

\subsection{Power flux to the blades}

The subfigure set (d) displays the surface temperature, the power flux density and the energy density flowing to the blade. The temperature is the basic signal which has been measured by the IR scanner. We have evaluated the temperature variation always at the centre of the blade near the tangency point of the plasma; this analysis provides the maximum temperature variation on the blade. The data were evaluated very densely (i.e. from line to line) around the times of disruptive heat flux and fewer data points were taken in the smooth part of the discharge and afterwards. The distribution of the analysed surface data points is shown in the bottom of figure set (c). The temperature data serve as input data for a 1-D heat transport code. The applied 1-D heat diffusion code requires surface temperature input data equally spaced in time. In addition, these data must span a time duration of several heat propagation times through the graphite tile (of the order of seconds).

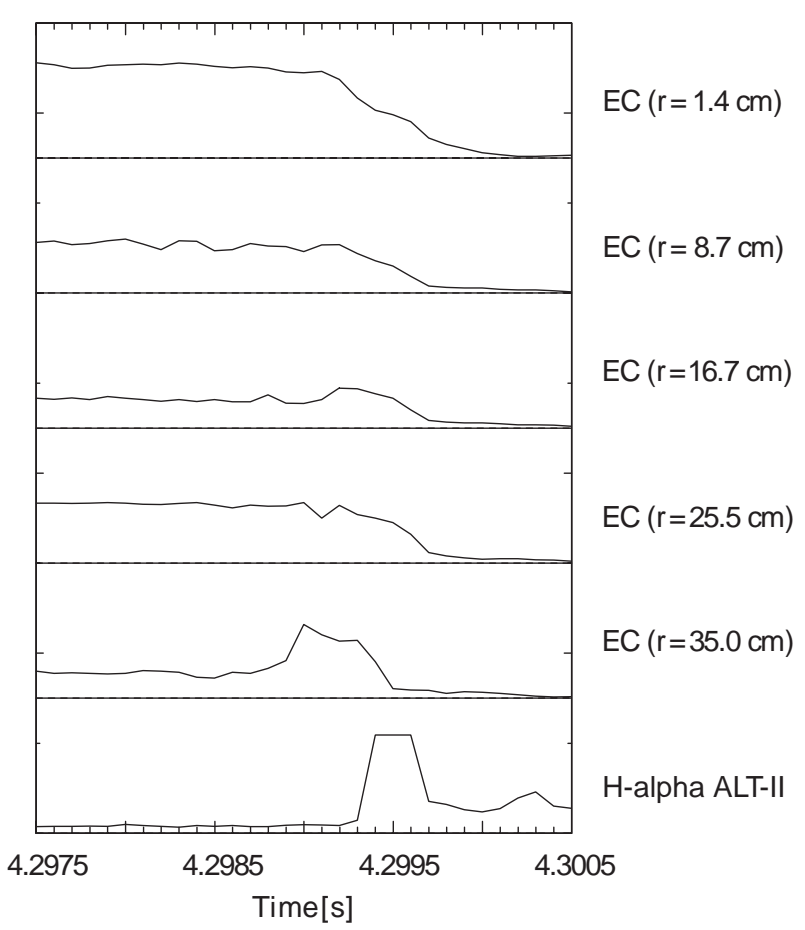

Figure 5(b). As Fig. 4(b) but for disruption shot 81523.

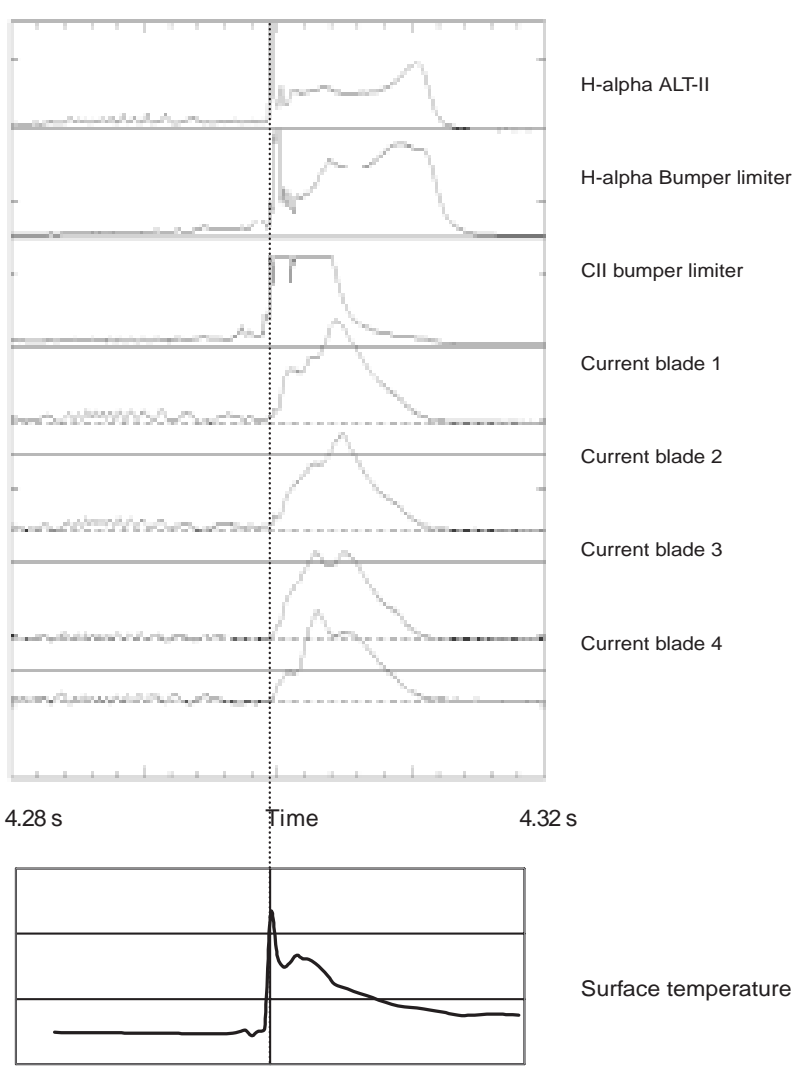

Figure 5(c). As Fig. 4(c) but for disruption shot 81523. 


\section{K.H. Finken et al.}

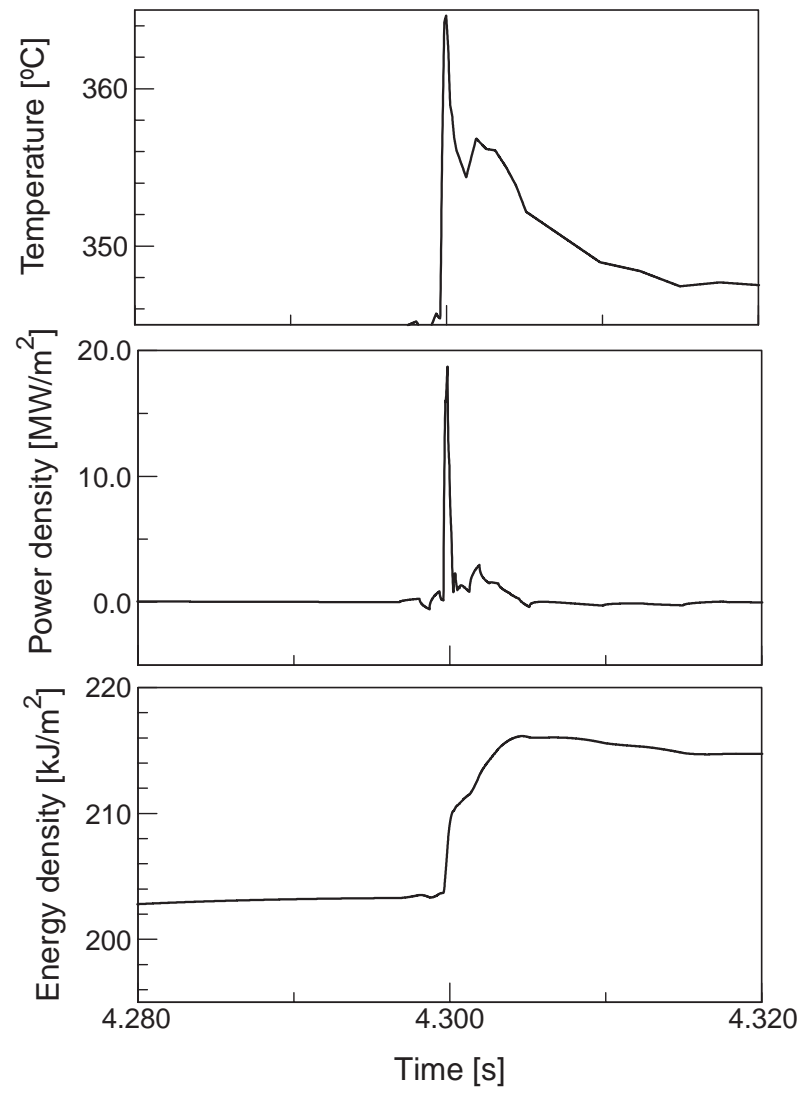

Figure 5(d). As Fig. 4(d) but for disruption shot 81523.

The data from the IR scanner, which are individually analysed by hand, are not equally spaced in time because the scanning frequency of both mirrors is not constant. Therefore a program has been written for creating the fitting input data structure. The time spacing of the input data points was selected to $10 \mu \mathrm{s}$ in order to reproduce the fast temperature rise during the disruptions.

The top trace of figure set (d) shows the well reproduced temperature trace (compare the bottom trace of (c) as 'original' data). The outcome of the analysis is the power density, plotted as the middle trace in figure set $(\mathrm{d})$. The heat pulse shows very distinct peaks at the occurrence of the minor or major disruptions. At all other times, its value is relatively small. Short overshoots to negative power values are probably due to imperfections of the input data and because heat is flowing also poloidally away from the most heated part (which we have analysed) and this is seen as negative power flux. Since the negative values are relatively small, we have not further optimized the 1-D code. As already stated in the discussion on the temporal and spatial deposition patterns, the power density during disruptions reaches several

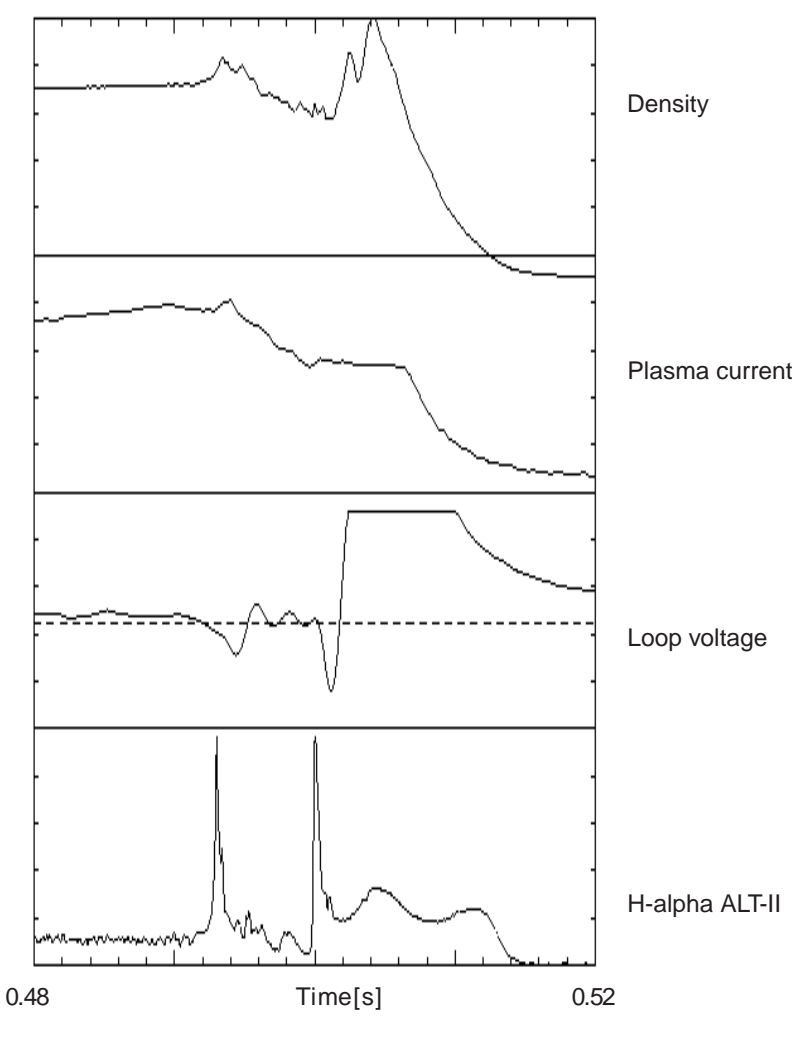

Figure 6(a). As Fig. 4(a) but for disruption shot 81540.

$10 \mathrm{MW} / \mathrm{m}^{2}$. This power flux is about $10^{3}-10^{4}$ times higher than the average power flux.

The energy density (lowest trace of figure set (d)) to the limiter is the integrated power density. The deposited energy density amounts to about $10 \mathrm{~kJ} / \mathrm{m}^{2}$ and is not particularly high; the same deposited energy is obtained after a few hundred milliseconds of blade exposure to the plasma. The power density is so enormously high only because the duration of the power pulse is so very short $(10-100 \mu \mathrm{s})$.

To obtain not only the power and energy density but the total amount of these quantities, we have to estimate the effective area where the heat pulse hits. The toroidal length of the ALT-II limiter amounts to $12 \mathrm{~m}$; we estimate the effective poloidal width on which the heat is deposited to be $8-15 \mathrm{~cm}$, which results in an effective area of roughly $1-2 \mathrm{~m}^{2}$.

The loss of internal energy over a disruption is on the average $50-80 \mathrm{~kJ}$; the diamagnetic loop signal has no good time resolution and therefore it is not meaningful to correlate it with the other signals. If we assume that an equal amount of the energy is radiated over the disruption and distributed on the outboard ALT-II limiter and the inner bumper limiter, we arrive at rather consistent results. The 


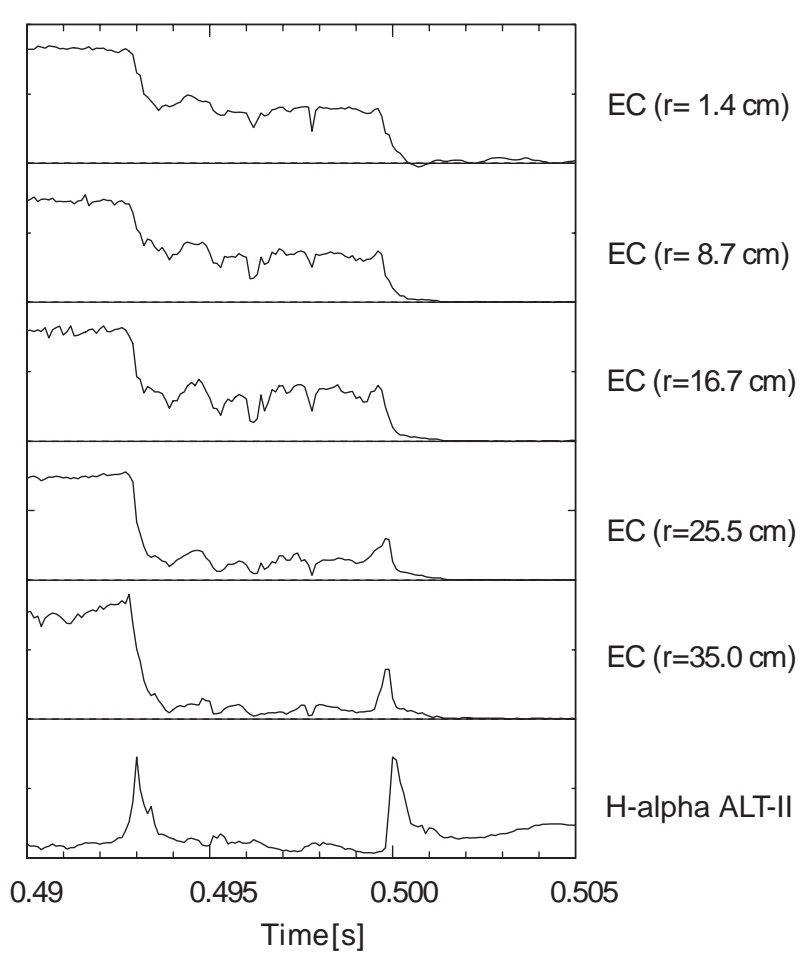

Figure 6(b). As Fig. 4(b) but for disruption shot 81540 . The ECE temperature range of the top channels is now $2 \mathrm{keV}$.

variation of the power density from disruption to disruption would roughly correspond to a peaking factor of three if we assume that the same fraction of power is always deposited on the whole ALT-II surface.

\section{Discussion}

The enhanced heat flux near the tangency line to the limiter, which we have characterized by the short power decay length $\lambda_{p \text {-short }}$, is a very persistent feature for all plasma conditions on TEXTOR-94: it has been observed in ohmic discharges, in L mode discharges, in RI mode discharges with the strong radiative edge cooling and finally even in disruptive heat pulses. In Fig. 3 many different edge plasma conditions are shown but nevertheless the effect is always present and the power fraction in the 'short decay length part' is always substantial and amounts to roughly $50 \%$. The 'short power decay length' effect was first described on TEXTOR-94 [1]; ALT-II probably had to have a very suitable tile shape in order to discover the effect. Nevertheless, other limiter tokamaks also showed a similar enhancement of the heat flux close to the last closed flux surface. This effect was, for example, called 'funnelling' [56, 57] at TFTR

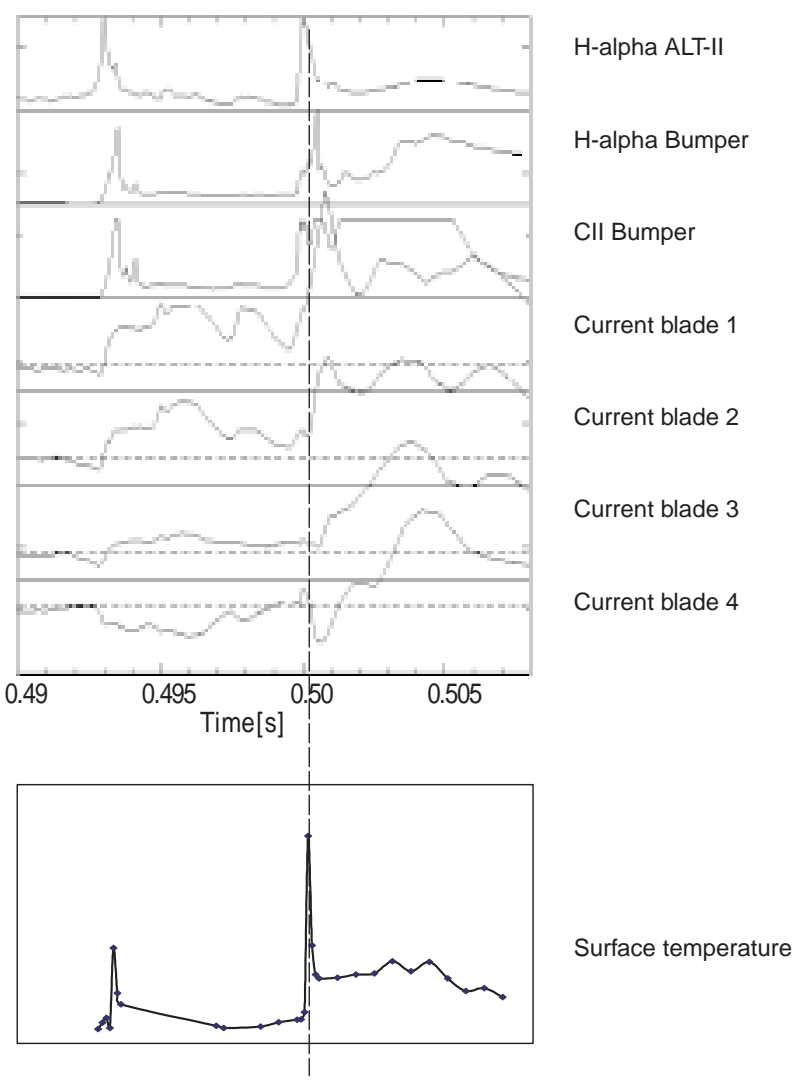

Figure 6(c). As Fig. 4(c) but for disruption shot 81540.

or 'sine-low' at Tore Supra [4] and may be based on the same physics as the 'short power decay length' effect of TEXTOR-94. The focussed power deposition near the tangency line even during the disruptive power pulses may require special considerations for the next step device: during disruptions the power will most likely also hit protection limiters and therefore we expect the same focussing effect to occur there as we have observed on TEXTOR-94.

Another point of discussion is the question on the physics behind the short pulses depositing the power on the limiters during disruptions. We had already estimated in Section 4.2 that power flow is most likely no diffusive process. The short duration of the pulses, their very high power and the good alignment of the deposited power with respect to the magnetic field do not fit into a picture of diffusive transport. In this sense it does not make a difference if the reason for the enhanced diffusion is turbulence or braiding of the magnetic field structure. The braiding or ergodization of the magnetic field lines is often thought to be the reason for the enhanced transport during the disruptions. According to 


\section{K.H. Finken et al.}

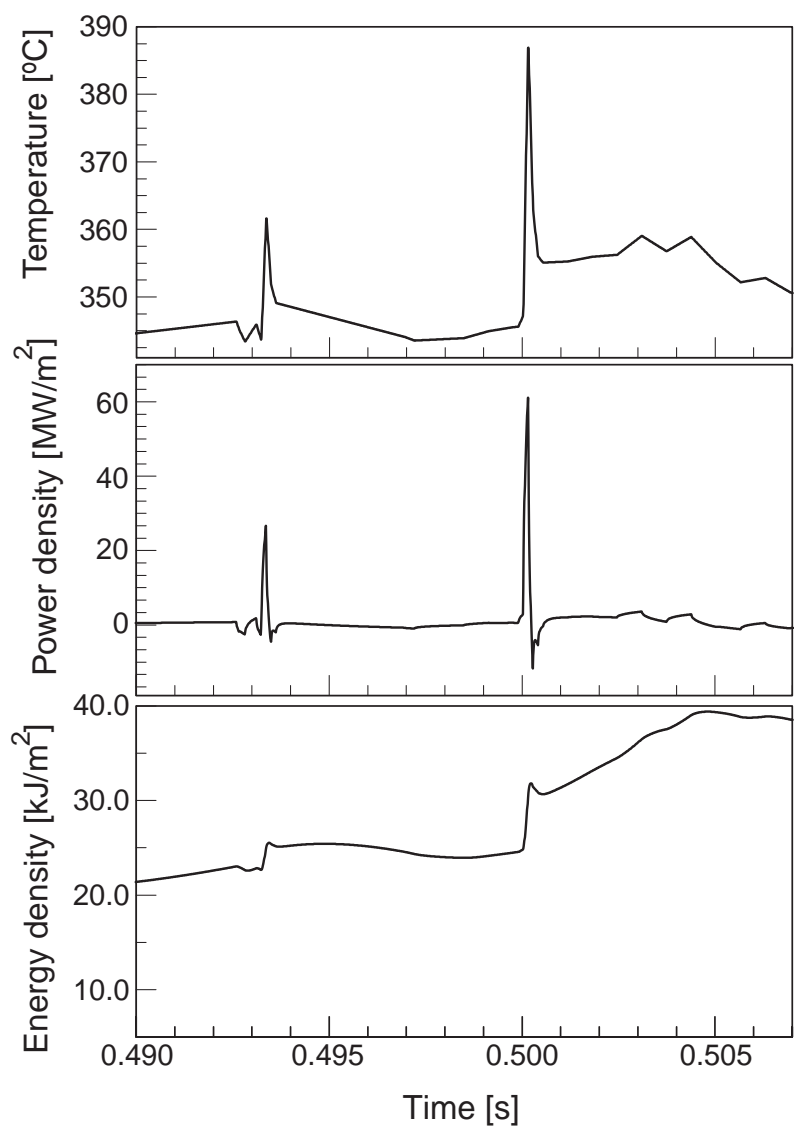

Figure 6(d). As Fig. 4(d) but for disruption shot 81540.

Rechester and Rosenbluth [58] magnetic perturbation with the radial field strength $B_{r}$ induces a diffusion $D$ of the order of

$D=\pi q R_{0}\left(\frac{B_{r}}{B}\right)^{2} v$

where $q$ is the safety factor, $R_{0}$ the major radius, $B \approx B_{t}$ the main (toroidal) magnetic field and $v$ the velocity of the component under consideration.

To obtain a value of $D=10^{2} \mathrm{~m}^{2} / \mathrm{s}$ as derived for $\kappa$ in Section 4.2, a perturbation field strength of about $0.02 \mathrm{~T}$ would be required (assuming $B=2.25 \mathrm{~T}$, $\left.v=10^{5} \mathrm{~m} / \mathrm{s}, q=3, R_{0}=1.75 \mathrm{~m}\right)$. This value of the perturbation field at the plasma edge is rather high and would provide a strong ergodization as the modelling of the Dynamic Ergodic Divertor [51] has shown. It is obvious that such a strong ergodization could destroy all 'good' magnetic surfaces in the tokamak and a discharge could not recover from this and will disrupt.

A particular property of ergodized edge magnetic fields has, to our knowledge, not been considered up to now within the framework of disruptions: it

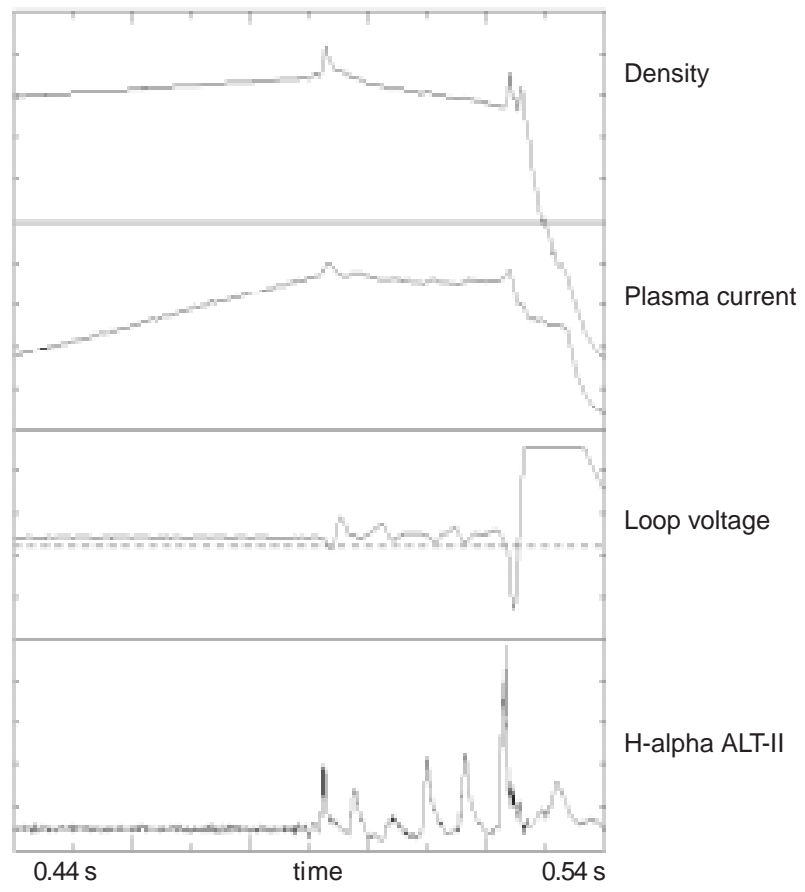

Figure 7(a). As Fig. 6(a) but for disruption shot 81533.

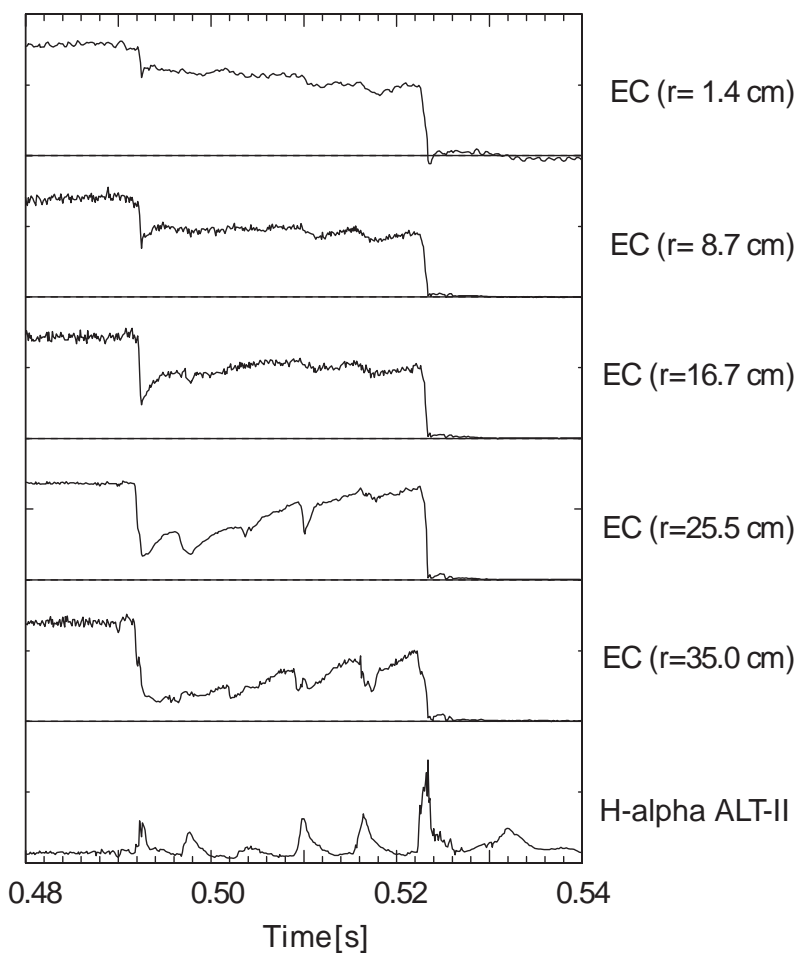

Figure 7(b). As Fig. 6(b) but for disruption shot 81533 .

is the laminar zone and we think that the properties of this laminar zone could be consistent with the observed temporal and spatial heat flux pattern. The 


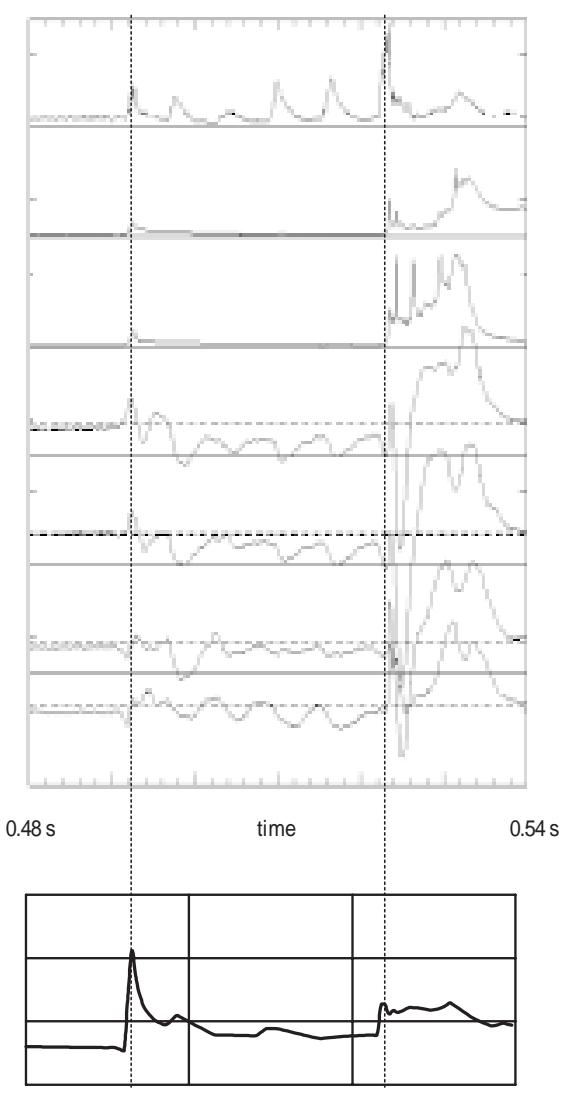

H-alpha ALT-II

H-alpha bumper

Cll bumper

Current blade 1

Current blade 2

Current blade 3

Current blade 4

Surface temperature

Figure 7(c). As Fig. 6(c) but for disruption shot 81533.

term 'laminar zone' has been introduced by the Tore Supra team [59] when discussing heat and particle fluxes of their ergodic divertor. It is a very general problem of open chaotic systems. The laminar zone is formed by those magnetic field lines which have a short connection length, i.e. field lines with one or a few poloidal turns between two intersections with material objects. We have shown [60] that the topology of the magnetic field lines in the laminar zone has a very high degree of order in contrast to that in the ergodic zone: during their relatively short path in between two wall intersections neighbouring magnetic field lines keep this property; if the underlying mode structure is relatively coarse - as expected during disruptions - field lines with similar properties form large coherent structures. For a perturbation field amplitude of a about a hundredth tesla at the plasma edge, these laminar field lines easily connect the edge plasma with the wall; the transport of heat and particles will be predominantly along the magnetic field lines and very similar to the transport in the normal scrape-off layer.

The scenario leading to the disruptive heat flux may develop as follows: at first internal modes
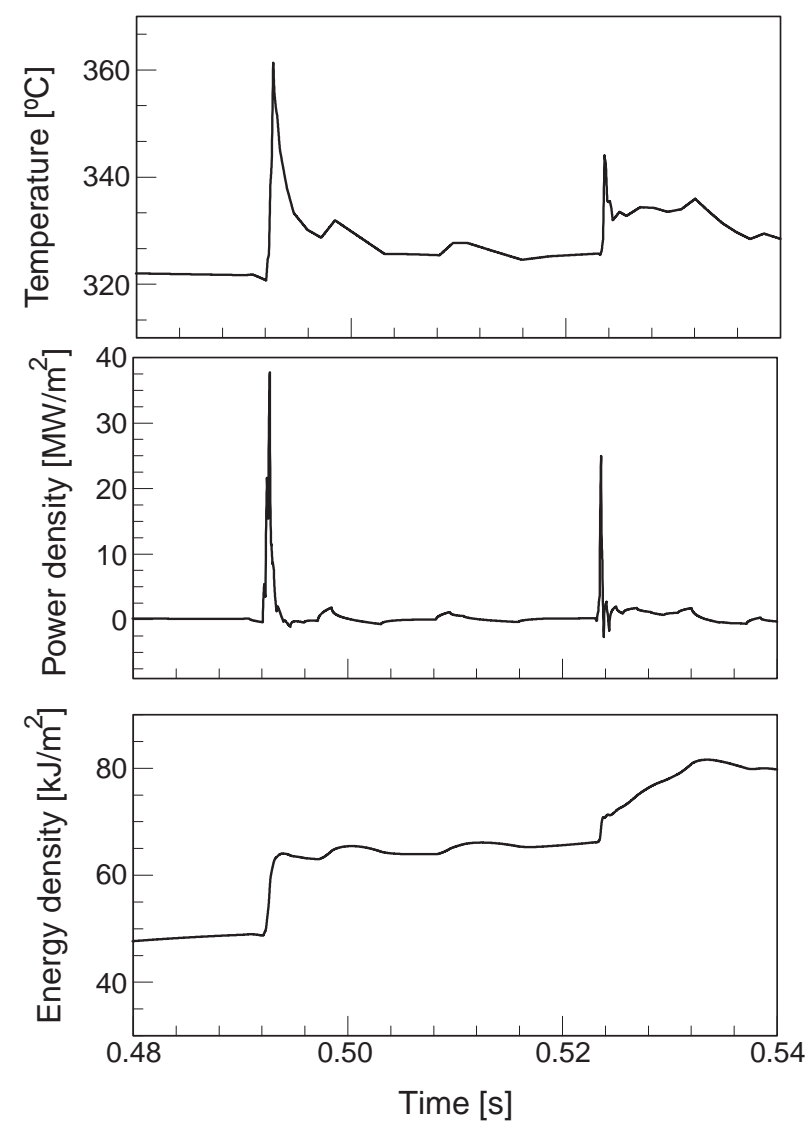

Figure 7(d). As Fig. 6(d) but for disruption shot 81533.

develop leading to an enhanced flow of energy from the core to the outer regions of the plasma. If the mode amplitude grows and reaches more and more the plasma edge, the laminar zone will grow. This growth and, connected with this process, the magnetic field line breaking and reconnection in the plasma edge can be sufficiently rapid [52] (e.g. it may have a time constant of $0.1 \mathrm{~ms}$ ). If the laminar zone has grown so much that it connects the energy reservoir at the plasma edge with the limiters or wall components, the rapid pulsed shaped loss of energy would be expected. In addition, the ergodized plasma structure is in general very complicated: it can contain 'sticky regions', isolated islands filled with hot plasma and other structures. If the growing laminar zone successively opens these reservoirs and shortcuts them to the wall components, an outflow of structured heat pulses in time would result, just as it is observed in the experiments. Since the connection length in the laminar zone amounts to only some $10 \mathrm{~m}$, a smearing out of the heat pulses is not expected, again in agreement with the observations. 


\section{References}

[1] Denner, T., et al., Nucl. Fusion 39 (1999) 83.

[2] Denner, T., Rev. Sci. Instru. 70 (1999) 68.

[3] Seigneur, A., et al., in Controlled Fusion and Plasma Physics (Proc. 20th Eur. Conf. Lisbon, 1993), Vol. 17C, Part II, European Physical Society, Geneva (1993) 603.

[4] Guilhem, D., et al., in Controlled Fusion and Plasma Physics (Proc. 24th Eur. Conf. Berchtesgaden, 1997), Vol. 21A, Part I, European Physical Society, Geneva (1997) 205.

[5] Stangeby, P.C., Pitcher, C.S., Elder, J.D., Nucl. Fusion 32 (1992) 2079.

[6] Matthews, G.F., Hill, D.N., Mahdavi, M.A., Nucl. Fusion 31 (1991) 1383.

[7] Ciotti, M., et al., in Controlled Fusion and Plasma Physics (Proc. 24th Eur. Conf. Berchtesgaden, 1997), Vol. 21A, Part IV, European Physical Society, Geneva (1997) 1749.

[8] Finken, K.H., et al., J. Nucl. Mater. 196-198 (1992) 220.

[9] Finken, K.H., et al., Nucl. Fusion 32 (1992) 915.

[10] Gruber, O., et al., Plasma Phys. Control. Fusion 35 (1993) B191.

[11] Pautasso, G., et al., Nucl. Fusion 34 (1994) 455.

[12] Wesson, J.A., et al., Nucl. Fusion 29 (1989) 641.

[13] Wesson, J.A., Ward, D.J., Rosenbluth, M.N., Nucl. Fusion 30 (1990) 1011.

[14] Waimann, G., Kuang, G., Nucl. Fusion 32 (1992) 645.

[15] Schüller, F.C., et al., in Controlled Fusion and Plasma Physics (Proc. 21st Eur. Conf. Montpellier, 1994), Vol. 18B, Part I, European Physical Society, Geneva (1994) 230.

[16] Savrukin, P.V., et al., Nucl. Fusion 34 (1994) 317.

[17] Taylor, P.L., et al., in Controlled Fusion and Plasma Physics (Proc. 22nd Eur. Conf. Bournemouth, 1995), Vol. 19C, Part IV, European Physical Society, Geneva (1995) 49.

[18] Yoshino, R., et al., J. Nucl. Mater. 220-222 (1995) 132 .

[19] Borras, K., Nucl. Fusion 31 (1991) 1035.

[20] Campbell, D.J., et al., in Controlled Fusion and Plasma Physics (Proc. 21st Eur. Conf. Montpellier, 1994), Vol. 18B, Part I, European Physical Society, Geneva (1994) 2.

[21] Campbell, D.J., et al., in Plasma Physics and Controlled Nuclear Fusion Research 1986 (Proc. 11th Int. Conf. Kyoto, 1986), Vol. 1, IAEA, Vienna (1987) 433.

[22] Frederickson, E.D., et al., Nucl. Fusion 33 (1993) 141.

[23] Greenwald, J.L., et al., Nucl. Fusion 28 (1988) 2199.

[24] Marinak, M.M., Nucl. Fusion 35 (1995) 399.
[25] Janos, A.C., et al., in Plasma Physics and Controlled Nuclear Fusion Research 1992 (Proc. 14th Int. Conf. Würzburg, 1992), Vol. 1, IAEA, Vienna (1993) 527.

[26] Nagayama, Y., et al., in Plasma Physics and Controlled Nuclear Fusion Research 1988 (Proc. 12th Int. Conf. Nice, 1988), Vol. 1, IAEA, Vienna (1989) 433.

[27] Remkes, G.J.J., Schüller, F.C., Plasma Phys. Control. Fusion 37 (1991) 181.

[28] Rommers, J.H., et al., in Controlled Fusion and Plasma Physics (Proc. 20th Eur. Conf. Lisbon, 1993), Vol. 17C, Part I, European Physical Society, Geneva (1994) 275.

[29] Ward, D.J., Wesson, J.A., Nucl. Fusion 32 (1992) 1117.

[30] Schuller, F.C., Plasma Phys. Control. Fusion 37 (1995) A135.

[31] Furth, H.P., Killeen, J., Rosenbluth, M.N., Phys. Fluids 6 (1963) 459.

[32] Bondeson, A., et al., Nucl. Fusion 31 (1991) 1695.

[33] Callen, J.D., et al., in Plasma Physics and Controlled Nuclear Fusion Research 1978 (Proc. 7th Int. Conf. Innsbruck, 1978), Vol. 1, IAEA, Vienna (1979) 415.

[34] Huysmans, G.Z.A., et al., in Controlled Fusion and Plasma Physics (Proc. 22nd Eur. Conf. Bournemouth, 1995), Vol. 19C, Part I, European Physical Society, Geneva (1995) 201.

[35] Smeulders, P., et al., ibid., Part IV, p. 61.

[36] Strait, E.J., et al., Phys. Rev. Lett. 62 (1989) 1282.

[37] Troyon, F., et al., Plasma Phys. Control. Fusion 26 (1984) 209.

[38] White, R.B., et al., Phys. Fluids 20 (1977) 800.

[39] Zohm, H., et al., Plasma Phys. Control. Fusion 37 (1995) A313.

[40] Fitzpatrick, R., Nucl. Fusion 33 (1993) 1049.

[41] Fitzpatrick, R., Phys. Plasmas 1 (1994) 3308.

[42] Fitzpatrick, R., Hender, T.C., Phys. Fluids B 3 (1991) 644.

[43] Hender, T.C., et al., Nucl. Fusion 32 (1992) 2091.

[44] Morris, A.W., et al., Phys. Fluids B 4 (1992) 413.

[45] Gvozdkov, Yu.V., Ivanov, N.V., Kakurin, A.M., Sov. J. Plasma Phys. 6 (1980) 130.

[46] Ivanov, N.V., Kakurin, A.M., Sov. J. Plasma Phys. 6 (1980) 22.

[47] Mazur, S., Phys. Plasmas 1 (1994) 3356.

[48] La Haye, R.J., et al., Phys. Fluids B 4 (1992) 2098.

[49] Fitzpatrick, R., Jensen T.H., Phys. Plasmas $\mathbf{3}$ (1996) 2641.

[50] Savrukhin, P., et al., IEEE Trans. Nucl. Sci. 43 (1996) 238.

[51] Finken, K.H. (Ed.), Fusion Eng. Des. (Special Issue) 37 (1997) 335.

[52] Finken, K.H., Nucl. Fusion 39 (1999) 707.

[53] Conn, R.W. (Ed.), Fusion Eng. Des. (Special Issue) 13 (1990) 251. 
[54] Messiaen, A.M., et al., Phys. Plasmas 4 (1997) 1690.

[55] Mioduszewski, P., J. Nucl. Mater. 111\&112 (1982) 253.

[56] Pitcher, C.S., et al., J. Nucl. Mater. 196-198 (1992) 241.

[57] Stangeby, P.C., Pitcher, C.S., Elder, J.D., Nucl. Fusion 32 (1992) 2079.

[58] Rechester, A.B., Rosenbluth, M.N., Phys. Rev. Lett. 40 (1978) 38.

[59] Ghendrih, O., Grosman, A., Capes, H., Plasma Phys. Control. Fusion 38 (1996) 1653.

[60] Finken, K.H., Eich, T., Kaleck, A., Nucl. Fusion 38 (1998) 515.
(Manuscript received 29 April 1999

Final manuscript accepted 24 November 1999)

E-mail address of K.H. Finken:

k.h.finken@fz-juelich.de

Subject classification: I2, Te 